\title{
SỬ DỤNG PHƯƠNG PHÁP HIỆ CHỈNH TOÁN HỌC CÁC TRI ĐO KÉP TRONG VIẸC KIỂM TRA VÀ HIẸU CHỈNH SAI SỐ HÊ THỐNG TRONG HAI DÃY ĐỘ CAO CHUẨN TOÀN CẦU VÀ Độ CAO CHUẨN QUỐC GIA
}

\author{
HÀ MINH HOÀ \\ Viện Khoa học Đo đạc và Bản đồ
}

\section{Tóm tắt:}

Hai dãy các độ cao chuẩn toàn cầu và độ cao chuẩn quốc gia trên các điểm GPS/thủy chuẩn được sử dụng để xác định thế trọng trường $W_{0}$ của mặt geoid cục bộ. Bài báo khoa học này trình bày các kết quả sử dụng phương pháp hiệu chỉnh hiệu trị đo kép để phát hiện, hiệu chỉnh sai số hệ thống trong hai dãy giá trị nêu trên và đề xuất tiêu chí xác định dãy giá trị bị sai số. Các kết quả thử nghiệm trên 17 điểm độ cao hạng I có đo GPS đã xác nhận sự hiệu quả của phương pháp được nghiên cứu.

\section{1. Đặt vấn đề}

Với mục đích xác định thế trọng trường của mặt geoid cục bộ Hòn Dấu (hoặc độ cao $H_{0}$ của mặt geoid cục bộ Hòn Dấu so với mặt geoid toàn cầu và cũng là độ cao của mặt quasigeoid cục bộ Hòn Dấu so với mặt quasigeoid toàn cầu), trong các tài liệu (Hà Minh Hòa, và nnk, 2012; Hà Minh Hòa, Nguyễn Bá Thủy, Phan Trọng Trịnh và nnk, 2016) đã sử dụng các dữ liệu sau đây trên các điểm độ cao hạng I: dữ liệu xử lý GPS (các tọa độ trắc địa $\bar{B}, \bar{L}, \bar{H}$ tương ứng với ellipsoid WGS-84 quốc tế); độ cao chuẩn $H^{\gamma}$ quốc gia; độ cao quasigeoid toàn cầu $\bar{\zeta}$ từ mô hình quasigeoid toàn cầu EGM2008 đã được tổ chức NGA (Mỹ) quy chuyển từ ellipsoid Trái đất trung bình TFS2008 về mặt ellipsoid WGS-84 quốc tế (để tiện sử dụng mô hình quasigeoid toàn cầu EGM2008 tương ứng với ellipsoid WGS84 quốc tế được gọi là mô hình quasigeoid toàn cầu EGM2008 - WGS84).

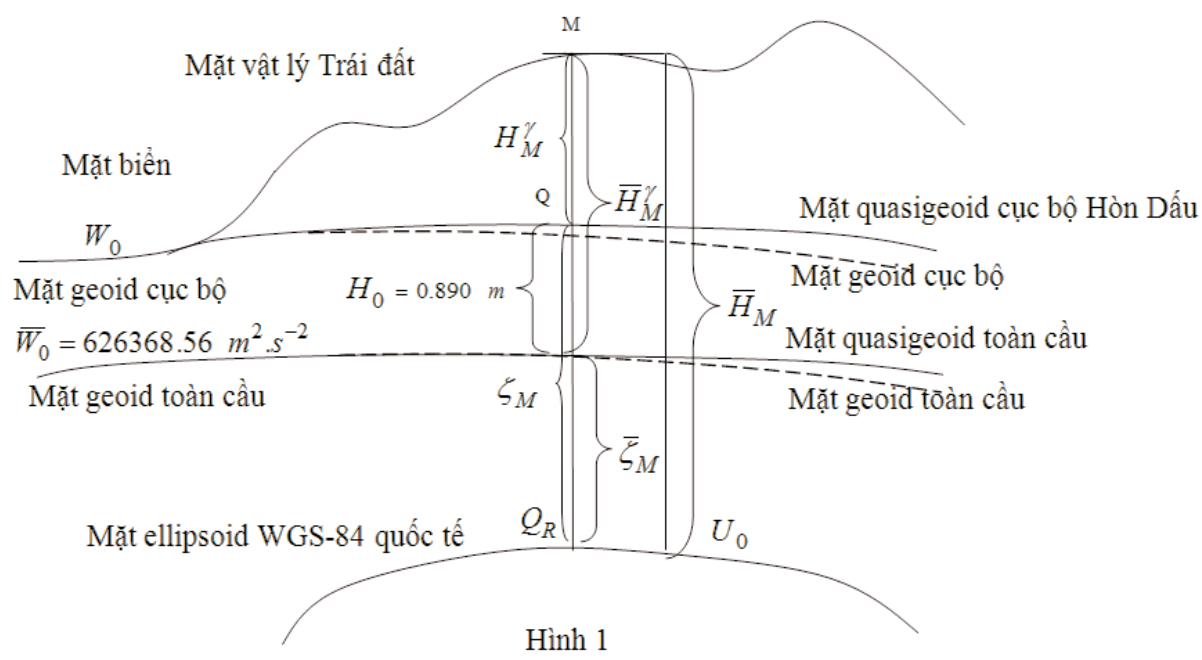

Ngày nhận bài: 12/10/2017, ngày chuyển phản biện: 19/10/2017, ngày chấp nhận phản biện: 25/10/2017, ngày chấp nhận đăng: 01/11/2017 
Trên hình 1, đối với điểm độ cao hạng I được ký hiệu là $\mathrm{M}: H_{M}^{r}$ - độ cao chuẩn hạng I trong hệ độ cao quốc gia HP72 tương ứng với mặt quasigeoid cục bộ Hòn Dấu;

$\bar{H}_{M}$ - độ cao trắc địa toàn cầu tương ứng với ellipsoid WGS-84 quốc tế và được xác định bằng công nghệ GPS; $\bar{\zeta}_{M}$ - độ cao quasigeoid toàn cầu được xác định từ mô hình quasigeoid toàn cầu EGM2008 - WGS84, thêm vào đó các giá trị nêu trên được chuyển về hệ triều 0 theo quy định quốc tế.

Trong các tài liệu (Hà Minh Hòa, và nnk, 2012; Hà Minh Hòa, Nguyễn Bá Thủy, Phan Trọng Trịnh và nnk, 2016), việc sử dụng các độ cao trắc địa toàn cầu $\mathscr{I}$ độ cao chuẩn quốc gia $H^{\gamma}$ và độ cao quasigeoid toàn cầu $\bar{\zeta}$ đã được chuyển về hệ triều 0 trên các điểm độ cao hạng I nhằm hai mục đích:

Mục đích 1: Đánh giá thế trọng trường $W_{0}$ của mặt geoid cục bộ Hòn Dấu (hoặc độ cao $H_{0}$ của mặt geoid cục bộ Hòn Dấu so với mặt geoid toàn cầu và cũng là độ cao của mặt quasigeoid cục bộ Hòn Dấu so với mặt quasigeoid toàn cầu);

Mục đích 2: Xác định quan hệ giữa mặt quasigeoid cục bộ Hòn Dấu và mặt quasigeoid toàn cầu để xây dựng mô hình quasigeoid cục bộ VIGAC2014 và đánh giá độ chính xác của mô hình này.

Để đạt mục đích 1 , thế trọng trường $W_{0}$ của mặt geoid cục bộ Hòn Dấu được xác định từ việc giải hệ phương trình số cải chính

$$
v_{i}=-W_{0}+\left(\bar{W}_{0}-y_{i}\right)(i=1,2, . ., n)
$$

theo điều kiện $\sum_{i=1}^{n} v_{i}^{2}=\min$ của phương pháp bình phương nhỏ nhất, ở đây $\mathrm{n}$ - tổng số các điểm độ cao hạng I được sử dụng; $\bar{W}_{0}=62636856.00 \mathrm{~m}^{2} \cdot \mathrm{s}^{-2}$ - thế trọng trường của mặt geoid toàn cầu; đại lượng $y_{i}=\bar{\gamma}_{i} \cdot d_{i} ; \bar{\gamma}_{i}$ - giá trị trung bình của gia tốc lực trọng trường chuẩn tại điểm i được xác định theo công thức:

$$
\bar{\gamma}_{i}=\gamma_{0}-0.000001534 . H_{i}^{\gamma}-0.000000000000036 .\left(H_{i}^{\gamma}\right)^{2}<m . s^{-2>},
$$

còn giá trị $y_{0}$ (đơn vị m. $\mathrm{s}^{-2}$ ) đối với ellipsoid WGS84 được xác định theo công thức:

$$
\gamma_{0}=9,7803253359 .\left(1+0,00530248 \cdot \sin ^{2} B-0,0000058497 \cdot \sin ^{2} 2 B\right)<m s^{-2}>,
$$

$d_{i}$ - hiệu giữa độ cao chuẩn toàn cầu $\bar{H}_{i}^{\gamma}$ và độ cao chuẩn quốc gia $H_{i}^{\gamma}$ được xác định theo công thức:

$$
d_{i}=\bar{H}_{i}^{\gamma}-H_{i}^{\gamma},
$$

thêm vào đó độ cao chuẩn toàn cầu được xác định theo công thức:

$$
\bar{H}_{i}^{\gamma}=\bar{H}_{i}-\bar{\zeta}_{i}
$$

Sau khi đã xác định được thế trọng trường $W_{0}$ của mặt geoid cục bộ Hòn Dấu, để đạt được mục đích 2, mối quan hệ giữa mặt quasigeoid cục bộ Hòn Dấu và mặt quasigeoid 
toàn cầu được thiết lập theo công thức:

$$
\zeta=\bar{\zeta}+H_{0}
$$

ở đây $\zeta$ - độ cao quasigeoid cục bộ Hòn Dấu; $H_{0}$ - độ cao của mặt quasigeoid cục bộ Hòn Dấu và mặt quasigeoid toàn cầu và được xác định theo công thức:

$$
H_{0}=\frac{\bar{W}_{0}-W_{0}}{\bar{\gamma}} \text {. }
$$

Các kết quả nghiên cứu trong các tài liệu (Hà Minh Hòa, và nnk, 2012; Hà Minh Hòa, Nguyễn Bá Thủy, Phan Trọng Trịnh và nnk, 2016) cho thế trọng trường của mặt geoid cục bộ Hòn Dấu $W_{0}=62636847,291 \mathrm{~m}^{2} \cdot \mathrm{s}^{-2}$; độ cao $H_{0}=0,890 \mathrm{~m}$ thêm vào đó sự không đổi của đại lượng $H_{0}$ trên lãnh thổ Việt Nam và toàn cầu đã được nghiên cứu trong nhiều tài liệu, ví dụ Hà Minh Hòa, và nnk, 2012; Hà Minh Hòa, Nguyễn Thị Thanh Hương, Lương Thanh Thạch, 2015; Nguyễn Tuấn Anh, 2015; Hà Minh Hòa, 2016.

Khi ký hiệu $\hat{\zeta}_{i}=\bar{H}_{i}-H_{i}^{\gamma}$ là độ cao quasigeoid GPS/thủy chuẩn trên điểm độ cao i, lưu ý (3) và đặt (4) vào (2) chúng ta nhận được hiệu:

$$
\bar{d}_{i}=d_{i}-H_{0}=\hat{\zeta}_{i}-\zeta_{i}
$$

là độ chênh của độ cao quasigeoid GPS/thủy chuẩn so với độ cao quasigeoid cục bộ $\zeta_{i}$ (4) trên điểm độ cao $\mathrm{i}$, ở đây hiệu $\bar{d}_{i}=d_{i}-H_{0}=d_{i}-0,890 \mathrm{~m} \quad$ (5) được sử dụng để đánh giá độ chính xác của mô hình quasigeoid cục bộ VIGAC2014 được xây dựng dựa trên công thức (4) và mô hình quasigeoid toàn cầu EGM2008 - WGS84.

Để nhận được giá trị thế trọng trường $W_{0}$ của mặt geoid cục bộ Hòn Dấu từ việc giải hệ phương trình số cải chính (1) dưới điều kiện $\sum_{i=1}^{n} v_{i}^{2}=\min$ của phương pháp bình phương nhỏ nhất sao cho giá trị này gần nhất với giá trị thực của nó và có sai số trung phương nhỏ nhất, theo định lý Gauss - Markov, các hiệu $d_{j}, i=1,2, \ldots, n$, không được chứa sai số hệ thống và sai số thô (xem chứng minh định lý Gauss - Markov trong tài liệu Markuze Y.I., 1990).

Vấn đề khoa học được đặt ra là phải kiểm tra sự có mặt, tìm kiếm và hiệu chỉnh các trị đo có chứa các sai số hệ thống và sai số thô nhằm đạt được hai mục đích nêu ở trên. Khi đó giá trị thế trọng trường $W_{0}$ của mặt geoid cục bộ Hòn Dấu sẽ được xác định một cách tin cậy nhất. Đây cũng là mục đích của bài báo khoa học này.

\section{Giải quyết vấn đề}

Khi coi các độ cao chuẩn toàn cầu $\bar{H}_{i}^{\gamma}, i=1,2, \ldots, n$ và các độ cao chuẩn quốc gia $H_{i}^{\gamma}$ $i=1,2, \ldots, n$ là hai dãy "các trị đo" độc lập, thì các hiệu $d_{j}, i=1,2, \ldots, n$, chính là các hiệu của các trị đo kép. Khi đó, việc kiểm tra sự có mặt của sai số hệ thống $C$ trong các hiệu trên hoàn toàn được thực hiện nhờ phương pháp hiệu chỉnh các trị đo kép (Bonsakov, V. D., Gaidaev, P. A., 1977), theo đó đối với các hiệu $\bar{d}_{i}, i=1,2, . ., n$, được xác định theo công thức (5), nếu thỏa mãn điều kiện 


$$
\left|\sum_{i=1}^{n} \bar{d}_{i}\right| \geq 0.25 x \sum_{i=1}^{n}\left|\bar{d}_{i}\right|
$$

thì các hiệu $\bar{d}_{i}, i=1,2, . ., n$, chứa sai số hệ thống $\mathrm{C}$ và sai số hệ thống được xác định theo công thức:

$$
C=\frac{\sum_{i=1}^{n} \bar{d}_{i}}{n},
$$

và số hiệu chỉnh do sai số hệ thống được xác định theo công thức:

$$
\delta C=-C \text {. }
$$

Các hiệu $\widetilde{\widetilde{d}}_{i}, i=1,2, . ., n$, được loại bỏ sai số hệ thống có dạng:

$$
\widetilde{\bar{d}}_{i}=\bar{d}_{i}+\delta C \text {. }
$$

Chúng ta nhận thấy rằng quá trình kiểm tra, tìm kiếm sai số hệ thống $\mathrm{C}$ trong các hiệu $\bar{d}_{i}=d_{i}-H_{0}$ là quá trình lặp gắn liền với việc xác định độ cao $H_{0}$. Trong thực tế giá trị đầu tiên của độ cao $H_{0}$ được xác định nhờ 09 điểm cơ sở của mô hình VIGAC2014 (xem các tài liệu Hà Minh Hòa, 2014; Hà Minh Hòa, Nguyễn Bá Thủy, Phan Trọng Trịnh, Nguyễn Nguyên Cương, và nnk, 2016).

Trong trường hợp phát hiện được sự có mặt của sai số hệ thống $C$ trong các hiệu $\bar{d}_{i}=d_{i}-H_{0}$, lưu ý độ cao $H_{0}$ là đại lượng không đổi, khi so sánh các hiệu $d_{i}$ (2) và $\bar{d}_{i}=d_{i}-H_{0}$, nẩy sinh hỏi: Trong dãy độ cao chuẩn toàn cầu $\bar{H}_{i}^{\gamma}$ và dãy độ cao chuẩn quốc gia $H_{i}^{\gamma}$ dãy độ cao nào chứa sai số hệ thống $\mathrm{C}$ ?. Câu trả lời không có sẵn trong lý thuyết hiệu chỉnh các trị đo kép. Chúng ta sẽ tìm kiếm lời giải đáp cho câu hỏi. Từ (9) lưu ý (2) và (5) chúng ta có:

$$
\widetilde{\bar{d}}_{i}=\bar{H}_{i}^{\gamma}-H_{i}^{\gamma}+\delta C-H_{0} .
$$

Bây giờ chúng ta giả thiết rằng độ cao chuẩn toàn cầu $\bar{H}_{i}^{\gamma}$ có chứa sai số hệ thống $\mathrm{C}$ và tiến hành hiệu chỉnh độ cao chuẩn này theo công thức:

$$
\widetilde{\bar{H}}_{i}^{\gamma}=\bar{H}_{i}^{\gamma}+\delta C, i=1,2, . ., n .
$$

Khi đó công thức (10) được viết lại dưới dạng:

$$
\tilde{\bar{d}}_{i}=\tilde{\bar{H}}_{i}^{\gamma}-H_{i}^{\gamma}-H_{0} \text {. }
$$

Nếu giả thiết trên là đúng, thì hiệu $\widetilde{\bar{d}}_{i}(12)$ sẽ có giá trị rất nhỏ (nhỏ hơn giá trị $\bar{d}_{i}$ có chứa sai số hệ thống $\mathrm{C}$ ). Nếu giả thiết trên là sai, tức độ cao chuẩn quốc gia $H_{i}^{\gamma}$ chứa sai số hệ thống $\mathrm{C}$, thì khi ký hiệu độ cao chuẩn quốc gia dưới dạng $H_{i}^{\gamma}=\widetilde{H}_{i}^{\gamma}+C$, ở đây $\widetilde{H}_{i}^{\gamma}$ - giá trị độ cao chuẩn quốc gia không chứa sai số hệ thống (giá trị chính xác), lưu ý (8) và 
(12), hiệu $\widetilde{\bar{d}}_{i}(12)$ sẽ bằng $\widetilde{\bar{d}}_{i}=\bar{H}_{i}^{\gamma}-\widetilde{H}_{i}^{\gamma}-2 C-H_{0}$ và sẽ có giá trị rất lớn (lớn hơn 2 lần sai số hệ thống $\mathrm{C}$ ). Như vậy, khi hiệu chỉnh độ cao chuẩn toàn cầu $\bar{H}_{i}^{\gamma}$ theo công thức (11) và dựa vào độ lớn của các hiệu $\tilde{\bar{d}}_{i}(12)$ chúng ta sẽ xác định được: dãy độ cao chuẩn toàn cầu $\bar{H}_{i}^{\gamma}$ hay dãy độ cao chuẩn quốc gia $H_{i}^{\gamma}$ chứa sai số hệ thống $\mathrm{C}$.

Bằng phương pháp kiểm tra, tìm kiếm các trị đo có chứa sai số hệ thống được trình bày ở trên đã phát hiện được sai số hệ thống trong các độ cao trắc địa $\overline{Z I}$ được Cục Đo đạc và Bản đồ Việt Nam xác định bằng công nghệ GPS trong giai đoạn $2009-2010$ trên 17 điểm độ cao hạng I bao gồm I(BH-HN)26, I(BH-HN)33, I(BH-HN)39, I(BH-HN)42, I(BHHN)48, I(HN-VL)4-1, I(HN-VL)6-1, I(LS-HN)22, I(LS-HN)29, I(LS-HN)36, I(HP-NB)14A, $\mathrm{I}(\mathrm{HN}-\mathrm{HP}) 11 \mathrm{~A}, \mathrm{I}(\mathrm{HN}-\mathrm{HP}) 2 \mathrm{~A}, \mathrm{I}(\mathrm{HN}-\mathrm{HP}) 5, \mathrm{I}(\mathrm{HN}-\mathrm{HP}) 7, \mathrm{I}(\mathrm{HN}-\mathrm{VL}) 10 \mathrm{~A}$ và $\mathrm{I}(\mathrm{HN}-\mathrm{VL}) 28-1$. Riêng độ cao trắc địa tại điểm $\mathrm{I}(\mathrm{HN}-\mathrm{VL}) 28-1$ có chứa sai số hệ thống $\mathrm{C}=-0,454 \mathrm{~m}$ và đã được hiệu chỉnh. Trường hợp này không được nghiên cứu trong bài báo này do độ lớn của sai số hệ thống khác so với sai số hệ thống trong 16 điểm điểm độ cao hạng I đầu tiên.

Đối với 16 điểm hạng I đầu tiên, trong bảng 1 trình bày các độ cao chuẩn toàn cầu $\bar{H}_{i}^{\gamma}$ trong hệ triều 0 , trong bảng 2 trình bày các hiệu $\bar{d}_{i}, i=1,2, . ., 16$, được xác định theo công thức (5). (Xem bảng 1,2)

\section{Bảng 1}

\begin{tabular}{|c|c|c|c|c|}
\hline STT & Điểm & $\begin{array}{c}\text { Độ cao trắc địa } \bar{H}_{z} \text { trong hệ } \\
\text { triều } 0(\mathrm{~m})\end{array}$ & $\begin{array}{c}\text { Độ cao quasigeod toàn } \\
\text { cầu } \overline{\bar{\xi}_{\mathrm{g}}} \text { từ mô hình } \\
\text { EGM2008 trong hệ triều } \\
0(\mathrm{~m})\end{array}$ & $\begin{array}{c}\text { Độ cao chuẩn toàn cầu } \\
\bar{H}_{z}^{\gamma}=\bar{H}_{z}-\bar{\zeta}_{z}\end{array}$ \\
\hline 1 & $\mathrm{I}(\mathrm{BH}-\mathrm{HN}) 26$ & 1.075 & -29.426 & 30.501 \\
\hline 2 & $\mathrm{I}(\mathrm{BH}-\mathrm{HN}) 33$ & -14.653 & -29.211 & 14.558 \\
\hline 3 & $\mathrm{I}(\mathrm{BH}-\mathrm{HN}) 39$ & -16.056 & -28.902 & 12.846 \\
\hline 4 & $\mathrm{I}(\mathrm{BH}-\mathrm{HN}) 42$ & -19.656 & -28.648 & 8.992 \\
\hline 5 & $\mathrm{I}(\mathrm{BH}-\mathrm{HN}) 48$ & -18.783 & -28.327 & 9.544 \\
\hline 6 & $\mathrm{I}(\mathrm{HN}-\mathrm{VL}) 4-1$ & -22.442 & -27.868 & 5.426 \\
\hline 7 & $\mathrm{I}(\mathrm{HN}-\mathrm{VL}) 6-1$ & -23.038 & -27.575 & 4.537 \\
\hline 8 & $\mathrm{I}(\mathrm{LS}-\mathrm{HN}) 22$ & -13.468 & -27.472 & 14.004 \\
\hline 9 & I(LS-HN)29 & -22.865 & -27.823 & 4.958 \\
\hline 10 & I(LS-HN)36 & -19.571 & -28.099 & 8.528 \\
\hline 11 & $\mathrm{I}(\mathrm{HP}-\mathrm{NB}) 14 \mathrm{~A}$ & -24.208 & -26.401 & 2.193 \\
\hline 12 & $\mathrm{I}(\mathrm{HN}-\mathrm{HP}) 11 \mathrm{~A}$ & -22.837 & -26.887 & 4.050 \\
\hline 13 & $\mathrm{I}(\mathrm{HN}-\mathrm{HP}) 2 \mathrm{~A}$ & -19.765 & -28.153 & 8.388 \\
\hline 14 & $\mathrm{I}(\mathrm{HN}-\mathrm{HP}) 5$ & -22.340 & -27.872 & 5.532 \\
\hline 15 & $\mathrm{I}(\mathrm{HN}-\mathrm{HP}) 7$ & -23.037 & -27.589 & 4.552 \\
\hline 16 & $\mathrm{I}(\mathrm{HN}-\mathrm{VL}) 10 \mathrm{~A}$ & -20.987 & -27.105 & 6.118 \\
\hline
\end{tabular}


Bảng 2

\begin{tabular}{|c|c|c|c|c|}
\hline STT & Điểm & \begin{tabular}{|} 
Độ cao chuẩn $H_{z}^{\gamma}$ trong hệ \\
độ cao HP72 trong hệ triều 0
\end{tabular} & $d_{i}=\bar{H}_{i}^{\gamma}-H_{i}^{\gamma}$ & $\bar{d}_{i}=d_{i}-0,890 m$ \\
\hline 1 & $\mathrm{I}(\mathrm{BH}-\mathrm{HN}) 26$ & 29,368 & 1.133 & 0.243 \\
\hline 2 & $\mathrm{I}(\mathrm{BH}-\mathrm{HN}) 33$ & 13.102 & 1.456 & 0.566 \\
\hline 3 & $\mathrm{I}(\mathrm{BH}-\mathrm{HN}) 39$ & 11.385 & 1.461 & 0.571 \\
\hline 4 & $\mathrm{I}(\mathrm{BH}-\mathrm{HN}) 42$ & 7.558 & 1.434 & 0.544 \\
\hline 5 & $\mathrm{I}(\mathrm{BH}-\mathrm{HN}) 48$ & 7.974 & 1.570 & 0.680 \\
\hline 6 & $\mathrm{I}(\mathrm{HN}-\mathrm{VL}) 4-1$ & 3.956 & 1.461 & 0.571 \\
\hline 7 & $\mathrm{I}(\mathrm{HN}-\mathrm{VL}) 6-1$ & 3.066 & 1.471 & 0.581 \\
\hline 8 & $\mathrm{I}(\mathrm{LS}-\mathrm{HN}) 22$ & 12,674 & 1.330 & 0.440 \\
\hline 9 & $\mathrm{I}(\mathrm{LS}-\mathrm{HN}) 29$ & 3.556 & 1.402 & 0.512 \\
\hline 10 & I(LS-HN)36 & 7.039 & 1.489 & 0.599 \\
\hline 11 & $\mathrm{I}(\mathrm{HP}-\mathrm{NB}) 14 \mathrm{~A}$ & 0.868 & 1.325 & 0.435 \\
\hline 12 & $\mathrm{I}(\mathrm{HN}-\mathrm{HP}) 11 \mathrm{~A}$ & 2,758 & 1.292 & 0.402 \\
\hline 13 & $\mathrm{I}(\mathrm{HN}-\mathrm{HP}) 2 \mathrm{~A}$ & 6.828 & 1.560 & 0.670 \\
\hline 14 & $\mathrm{I}(\mathrm{HN}-\mathrm{HP}) 5$ & 3.938 & 1.594 & 0.704 \\
\hline 15 & $\mathrm{I}(\mathrm{HN}-\mathrm{HP}) 7$ & 3.046 & 1.506 & 0.616 \\
\hline 16 & $\mathrm{I}(\mathrm{HN}-\mathrm{VL}) 10 \mathrm{~A}$ & 4,764 & 1.354 & 0.464 \\
\hline
\end{tabular}

Chúng ta thấy từ bảng 2 rằng các hiệu $\bar{d}_{i}$ có giá trị khá lớn và có cùng dấu. $\sum_{i=1}^{16} \bar{d}_{i}=8.598 \mathrm{~m}$. Do điều kiện (6) $\left|\sum_{i=1}^{16} \bar{d}_{i}\right|=8.598 \geq 0.25 x \sum_{i=1}^{16}\left|\bar{d}_{i}\right|=2.150$ thỏa mãn, nên dãy các hiệu $\bar{d}_{i}, i=1,2, . ., 16$, chứa sai số hệ thống $C=\frac{\sum_{i=1}^{16} \bar{d}_{i}}{16}=0.537 \mathrm{~m}$.

Như vậy số hiệu chỉnh sai số hệ thống $\delta C=-0.537 \mathrm{~m}$.

Chúng ta giả thiết rằng các độ cao chuẩn toàn cầu $\bar{H}_{i}^{\gamma}, i=1,2, . ., 16$ chứa sai số hệ thống. Trong bảng 3 trình bày các độ cao chuẩn toàn cầu ${\widetilde{H_{i}}}_{i}^{\gamma}, i=1,2, . ., 16$ được hiệu chỉnh theo công thức (11) và trong bảng 4 trình bày các hiệu $\widetilde{\widetilde{d}}_{i}, i=1,2, . ., 16$, được tính toán theo công thức (12). (Xem bảng 3, 4)

So sánh các hiệu $\bar{d}$ trong bảng 2 và các hiệu 
Bảng 3

\begin{tabular}{|c|c|c|c|c|}
\hline STT & Điểm & $\begin{array}{c}\text { Độ cao chuẩn toàn cầu } \\
\bar{H}_{z}^{\gamma}=\bar{H}_{z}-\bar{\zeta}_{z} \\
(\mathrm{~m})\end{array}$ & $\begin{array}{c}\text { Số hiệu chỉnh } \\
\delta C=-0.537 m\end{array}$ & $\begin{array}{c}\text { Độ cao chuẩn toàn cầu } \\
\overline{\bar{H}}_{z}^{\gamma}=\bar{H}_{z}^{\gamma}+\delta C \\
\text { được hiệu chỉnh (m) }\end{array}$ \\
\hline 1 & $\mathrm{I}(\mathrm{BH}-\mathrm{HN}) 26$ & 30.501 & -0.537 & 29.964 \\
\hline 2 & $\mathrm{I}(\mathrm{BH}-\mathrm{HN}) 33$ & 30.501 & -0.537 & 29.964 \\
\hline 3 & $\mathrm{I}(\mathrm{BH}-\mathrm{HN}) 39$ & 14.558 & -0.537 & 14.021 \\
\hline 4 & $\mathrm{I}(\mathrm{BH}-\mathrm{HN}) 42$ & 12.846 & -0.537 & 12.309 \\
\hline 5 & $\mathrm{I}(\mathrm{BH}-\mathrm{HN}) 48$ & 8.992 & -0.537 & 8.455 \\
\hline 6 & $\mathrm{I}(\mathrm{HN}-\mathrm{VL}) 4-1$ & 9.544 & -0.537 & 9.007 \\
\hline 7 & $\mathrm{I}(\mathrm{HN}-\mathrm{VL}) 6-1$ & 5.426 & -0.537 & 4.889 \\
\hline 8 & $\mathrm{I}(\mathrm{LS}-\mathrm{HN}) 22$ & 4.537 & -0.537 & 4.000 \\
\hline 9 & $\mathrm{I}(\mathrm{LS}-\mathrm{HN}) 29$ & 14.004 & -0.537 & 13.467 \\
\hline 10 & $\mathrm{I}(\mathrm{LS}-\mathrm{HN}) 36$ & 4.958 & -0.537 & 4.421 \\
\hline 11 & $\mathrm{I}(\mathrm{HP}-\mathrm{NB}) 14 \mathrm{~A}$ & 8.528 & -0.537 & 7.991 \\
\hline 12 & $\mathrm{I}(\mathrm{HN}-\mathrm{HP}) 11 \mathrm{~A}$ & 2.193 & -0.537 & 1.656 \\
\hline 13 & $\mathrm{I}(\mathrm{HN}-\mathrm{HP}) 2 \mathrm{~A}$ & 4.050 & -0.537 & 3.513 \\
\hline 14 & $\mathrm{I}(\mathrm{HN}-\mathrm{HP}) 5$ & 8.388 & -0.537 & 7.851 \\
\hline 15 & $\mathrm{I}(\mathrm{HN}-\mathrm{HP}) 7$ & 5.532 & -0.537 & 4.995 \\
\hline 16 & $\mathrm{I}(\mathrm{HN}-\mathrm{VL}) 10 \mathrm{~A}$ & 6.118 & -0.537 & 5.581 \\
\hline
\end{tabular}

hiệu hiệu $\widetilde{\bar{d}}_{i}$ trong bảng 4 chúng ta thấy rằng $\left|\sum_{i=1}^{16} \tilde{\bar{d}}_{i}\right|=0,006<0.25 x \sum_{i=1}^{16}\left|\tilde{\bar{d}}_{i}\right|=0.25 x 1.458=0.364$. Do đó trong dãy các hiệu $\widetilde{\bar{d}}_{i}$ trong bảng 4 không còn chứa sai số hệ thống nữa và giả thiết về sự tồn tại sai số hệ thống trong dãy các độ cao toàn cầu $\bar{H}_{i}^{\gamma}, i=1,2, . ., 16$ được chấp nhận.

Trong các tài liệu (Hà Minh Hòa, và nnk, 2012; Hà Minh Hòa, Nguyễn Bá Thủy, Phan Trọng Trịnh và nnk, 2016), các độ cao toàn cầu được hiệu chỉnh $\widetilde{\bar{H}}_{i}^{\gamma}, i=1,2, . ., 16$ trong bảng 3 được sử dụng để lập hệ phương trình số cải chính (1), còn các hiệu được hiệu chỉnh $\widetilde{\bar{d}}_{i}, i=1,2, . ., 16$, được sử dụng để đánh giá độ chính xác của mô hình quasigeoid khởi đầu VIGAC2014.

Đến đây lại nẩy sinh câu hỏi: do trong dãy các độ cao toàn cầu $\bar{H}_{i}^{\gamma}, i=1,2, . ., 16$ chứa sai số hệ thống, vậy sai số hệ thống thuộc các độ cao trắc địa $\bar{H}_{i}, i=1,2, . ., 16$ hay thuộc các 
độ cao quasigeoid toàn cầu $\bar{\zeta}_{i}, i=1,2, . ., 16$ ?. Để trả lời câu hỏi này, bằng cách tương tự như trên, chúng ta làm như sau. Lưu ý công thức (3), chúng ta viết lại công thức (11) dưới dạng:

$$
\widetilde{\bar{H}}_{i}^{\gamma}=\bar{H}_{i}-\bar{\zeta}+\delta C, i=1,2, . ., n .
$$

Khi giả thiết rằng độ cao trắc địa $\bar{H}_{i}$ có chứa sai số hệ thống $C$ và tiến hành hiệu chỉnh độ cao trắc địa này theo công thức:

$$
\widetilde{\bar{H}}_{i}=\bar{H}_{i}+\delta C, i=1,2, . ., n,
$$

\section{Bảng 4}

\begin{tabular}{|c|c|c|}
\hline STT & Điểm & $\begin{array}{c}\text { Các hiệu } \\
\tilde{\bar{d}}_{i}=\tilde{\bar{H}}_{i}^{\gamma}-H_{i}^{\gamma}-0.890 ~ m \\
\text { được điều chỉnh (m) }\end{array}$ \\
\hline 1 & $\mathrm{I}(\mathrm{BH}-\mathrm{HN}) 26$ & -0.294 \\
\hline 2 & $\mathrm{I}(\mathrm{BH}-\mathrm{HN}) 33$ & 0.029 \\
\hline 3 & $\mathrm{I}(\mathrm{BH}-\mathrm{HN}) 39$ & 0.034 \\
\hline 4 & $\mathrm{I}(\mathrm{BH}-\mathrm{HN}) 42$ & 0.007 \\
\hline 5 & $\mathrm{I}(\mathrm{BH}-\mathrm{HN}) 48$ & 0.143 \\
\hline 6 & $\mathrm{I}(\mathrm{HN}-\mathrm{VL}) 4-1$ & 0.034 \\
\hline 7 & $\mathrm{I}(\mathrm{HN}-\mathrm{VL}) 6-1$ & 0.044 \\
\hline 8 & $\mathrm{I}(\mathrm{LS}-\mathrm{HN}) 22$ & -0.097 \\
\hline 9 & $\mathrm{I}(\mathrm{LS}-\mathrm{HN}) 29$ & -0.025 \\
\hline 10 & $\mathrm{I}(\mathrm{LS}-\mathrm{HN}) 36$ & 0.062 \\
\hline 11 & $\mathrm{I}(\mathrm{HP}-\mathrm{NB}) 14 \mathrm{~A}$ & -0.102 \\
\hline 12 & $\mathrm{I}(\mathrm{HN}-\mathrm{HP}) 11 \mathrm{~A}$ & -0.135 \\
\hline 13 & $\mathrm{I}(\mathrm{HN}-\mathrm{HP}) 2 \mathrm{~A}$ & 0.133 \\
\hline 14 & $\mathrm{I}(\mathrm{HN}-\mathrm{HP}) 5$ & 0.167 \\
\hline 15 & $\mathrm{I}(\mathrm{HN}-\mathrm{HP}) 7$ & 0.079 \\
\hline 16 & $\mathrm{I}(\mathrm{HN}-\mathrm{VL}) 10 \mathrm{~A}$ & -0.073 \\
\hline
\end{tabular}

công thức (12) được biểu diễn dưới dạng:

$$
\widetilde{\bar{H}}_{i}^{\gamma}=\widetilde{\bar{H}}_{i}-\bar{\zeta}, i=1,2, . ., n .
$$

Nếu giả thiết nêu trên là đúng, thì các giá trị độ cao chuẩn toàn cầu $\widetilde{\bar{H}}_{i}^{\gamma}$ được xác định theo các công thức (11) và (15) sẽ trùng nhau. Nếu giả thiết nêu trên là sai, tức các độ cao quasigeoid toàn cầu ç chứa sai số hệ thống $\mathrm{C}$, thì khi ký hiệu độ cao quasigeoid toàn cầu $\bar{\zeta}_{i}=\widetilde{\bar{\zeta}}_{i}+C$, ở đây $\bar{\zeta}_{i}$ - giá trị chính xác của độ cao quasigeoid toàn cầu (không chứa sai số hệ thống), lưu ý (8), (14) công thức (15) nhận giá trị

$$
\widetilde{\bar{H}}_{i}^{\gamma}=\bar{H}_{i}-\widetilde{\zeta}-2 C, i=1,2, . ., n,
$$

có sai số hệ thống rất lớn bằng $2 \mathrm{C}$.

Như vậy bằng cách sử dụng giả thiết rằng độ cao trắc địa $\bar{H}_{i}$ có chứa sai số hệ thống $\mathrm{C}$ và hiệu chỉnh các độ cao trắc địa theo công thức (14), chúng ta hoàn toàn có cơ sở khoa học để xác định: sai số hệ thống C thuộc các độ cao trắc địa $\bar{H}_{i}, i=1,2, . ., 16$ hay thuộc các độ cao quasigeoid toàn cầu $\bar{\zeta}_{i}, i=1,2, . ., 16$ Với mục đích xác định xem sai số hệ thống $\mathrm{C}$ thuộc các độ cao trắc địa $\bar{H}_{i}, i=1,2, . ., 16$ hay thuộc các độ cao quasigeoid toàn cầu $\bar{\zeta}_{i}, i=1,2, . ., 16$, trong các tài liệu (Hà Minh Hòa, và nnk, 2012; Hà Minh Hòa, Nguyễn Bá Thủy, Phan Trọng Trịnh và nnk, 2016) đã sử dụng giả thiết rằng độ cao trắc địa $\bar{H}_{i}$ có chứa sai số hệ thống $\mathrm{C}$ và hiệu chỉnh các độ cao trắc địa theo công thức (14). Do các giá trị độ cao chuẩn toàn cầu $\widetilde{\bar{H}}_{i}^{\gamma}$ được xác định theo các công thức (11) và (15) trùng nhau, nên giả thiết được sử dụng là đúng.

Vậy tại sao độ cao trắc địa nhận được từ các kết quả xử lý dữ liệu GPS bằng phần 
mềm GPSurvey có thể chứa sai số hệ thống ?. Trong tài liệu (Lau Ngoc Nguyen, Viet Tuan Duong, and Richard Coleman, 2016) đã thông báo các kêt quả đo GPS trên mạng lưới bao gồm 67 baselines độc lập tại Hải Dương vào năm 2014 và xử lý dữ liệu GPS bằng phần mềm TBC v 2.0 (phiên bản sau của GPSurvey). Kết quả xử lý GPS cho thấy một số baselimes có sai số hệ thống ở mức một vài $\mathrm{dm}$. Các nghiên cứu thử nghiệm trong tài liệu trên cho thấy trong điều kiện cấu hình vệ tinh không tốt, tầng điện ly biến thiên mạnh và ở một số điểm GPS ảnh hưởng của hiện tượng đa đường truyền lớn, phần mềm TBC v 2.0 không cho lời giải Fixed chính xác khi giải trị nguyên đa trị. Ngoài ra, việc xử lý các dữ liệu GPS để xác định các baselines với các chiều dài lớn hơn $30 \mathrm{~km}$ bằng phần mềm GPSurvey có thể gây ra sai số hệ thống là vấn đề cần phải được tiếp tục nghiên cứu rất nghiêm túc.

Trong các tài liệu (Hà Minh Hòa, và nnk, 2012; Hà Minh Hòa, Nguyễn Bá Thủy, Phan Trọng Trịnh và nnk, 2016) các hiệu $d_{i}=\bar{H}_{i}^{\gamma}-H_{i}^{\gamma}$ (2) còn được kiểm tra lần cuối khả năng chứa sai số hệ thống và sai số thô theo tiêu chuẩn Smirnov. Tuy nhiên, vấn đề này không thuộc nội dung nghiên cứu của bài báo này./.

\section{Kết luận}

Với việc phát triển mạnh mẽ các tiến bộ hiện đại của khoa học - kỹ thuật vào việc giải quyết các bài toán trắc địa vật lý đòi hỏi các nhà trắc địa phải sử dụng các công cụ hiện đại của Lý thuyết hiệu chỉnh toán học các dữ liệu trắc địa trong bối cảnh các nguồn dữ liệu trắc địa ngày càng đa dạng, phong phú nhằm phân tích, đánh giá, phát hiện và loại bỏ các sai số hệ thống và sai số thô trong các dữ liệu trắc địa, đặc biệt khi giải quyết các bài toán trắc địa vật lý theo phương pháp bình phương nhỏ nhất. Trong bài báo khoa học này đã sử dụng phương pháp hiệu chỉnh toán học các trị đo kép để đánh giá, phát hiện và loại bỏ các sai số hệ thống trong các dữ liệu xử lý GPS, độ cao chuẩn quốc gia và độ cao quasigeoid toàn cầu trên các điểm độ cao hạng I và phát triển các tiêu chí xác định dãy các trị đo chứa sai số này. Phương pháp được nghiên cứu đã được áp dụng hiệu quả để kiểm tra sự có mặt, tìm kiếm sai số hệ thống và hiệu chỉnh các độ cao chuẩn toàn cầu trên 17 điểm độ cao hạng I quốc gia phục vụ việc giải quyết bài toán xác định thế trọng trường $W_{0}$ của mặt geoid cục bộ Hòn Dấu và xây dựng mô hình quasigeoid quốc gia khởi đầu VIGAC2014.I.O

\section{Tài liệu tham khảo}

[1]. Большаков, В.Д., Гайдаев, П. А., 1977 Теория математической обработки геодезических измерений. Москва, Недра.

Bonsakov, V. D., Gaidaev, P. A., 1977. Lý thuyết hiệu chỉnh toán học các kết quả đo đạc trắc địa. Matxcơva, Nedra (Tiếng Nga).

[2]. Hà Minh Hòa, và nnk, 2012. Nghiên cứu cơ sở khoa học của việc hoàn thiện hệ độ cao gắn liền với việc xây dựng hệ tọa độ động lực quốc gia. Đề tài khoa học và công nghệ cấp Bộ Tài nguyên và Môi trường giai đoạn 2010 - 2012. Hà Nội - 2012.

[3]. Hà Minh Hòa, 2014. Lý thuyết và thực tiễn của Trọng lực trắc địa. Nhà xuất bản Khoa học và Kỹ thuật, 592 trg., Hà Nội - 2014.

[4]. Hà Minh Hòa, Nguyễn Thị Thanh Hương, Lương Thanh Thạch, 2015. Đánh giá kiểm 
tra thế trọng trường $W_{0}$ của mặt geoid cục bộ Hòn Dấu trên cơ sở sử dụng 89 điểm độ cao hạng I. Tạp chí Khoa học Đo đạc và Bản đồ, số 26, tháng 12/2015, trg. 1 - 15.

[5]. Hà Minh Hòa, 2016. Nghiên cứu sự thay đổi độ cao giữa mặt quasigeoid cục bộ Hòn Dấu và mặt quasigeoid toàn cầu trên phạm vi toàn cầu. Tạp chí Khoa học Đo đạc và Bản đồ, số 28, tháng 06/2016, trg. 1 - 7.

[6]. Hà Minh Hòa, Nguyễn Bá Thủy, Phan Trọng Trịnh, Nguyễn Nguyên Cương, Nguyễn Phi Sơn, Nguyễn Thị Thanh Hương, và nnk, 2016. "Nghiên cứu đánh giá các mặt chuẩn mực nước biển (mặt " 0 " độ sâu, trung bình và cao nhất) theo các phương pháp trắc địa, hải văn và kiến tạo hiện đại phục vụ xây dựng các công trình và quy hoạch đới bờ Việt Nam trong xu thế biến đổi khí hậu". Đề tài khoa học và phát triển công nghệ mã số KC.09.19/11 -15 giai đoạn $2012-2015$ thuộc Chương trình KH\&CN trọng điểm cấp Nhà nước KC-09/11-15 "Nghiên cứu khoa học và công nghệ phục vụ quản lý biển, hải đảo".

[7]. Lau Ngoc Nguyen, Viet Tuan Duong, and Richard Coleman, 2016. Validation of GNSS proceasing results from some commercial software packages under un - advantageous conditions. Geoinformatics for Natural Resources, Hazards and Sustainability. International Conference On Geolnformatics for Spatial - Infrastructurer Development in Earth \& Applied Sciences (GIS - IDEAS), ESASGD-2016 .

[8]. Маркузе Ю. И., 1990. Основы уравнительных вычислений. М.: Недра, 1990.-290 c. Markuze Y.I., 1990. Các cơ sở tính toán bình sai. M. Nedra, 1990, 290 trg. (Tiếng Nga).

[9]. Nguyễn Tuấn Anh, 2015. Nghiên cứu chi tiết độ cao của mặt geoid cục bộ Hòn Dấu so với mặt geoid toàn cầu trên lãnh thổ Việt Nam. Tạp chí Khoa học Đo đạc và Bản đồ, No25, 09/2015.O

\section{Summary}

Using of a method of mathematical processing of double meassurements for detecting and correcting systematic error in two series of global and national normal heights

\section{Ha Minh Hoa}

\section{Vietnam Institute of Geodesy and Cartography}

A two series of the global and national normal heights has been used for determination of geopotential $W_{0}$ of a local geoid. This scientific articale presents results of using of a method of mathematical processing of double meassurements for detecting and correcting systematic error in above mentioned two series and proposes criterion for determination of erroneous series. Experiment results on the $17 \mathrm{co}$ - located GPS observations first order benchmarks showed effectivity of researched method. $O$ 